Diet, exercise and dementia: The potential impact of a Mediterranean diet pattern and physical activity on cognitive health in a UK population

Running head: Mediterranean diet, physical activity and dementia

Keywords: Mediterranean diet, dementia, Alzheimer's disease, physical activity, RCT Randomised controlled trial

R. Bundy and A.M. Minihane

University of East Anglia, Norwich, UK

Correspondence: Dr Rafe Bundy, Research Fellow, Norwich Medical School, Bob Champion

Research and Education (BCRE) Building, James Watson Road, University of East Anglia, Norwich Research Park, Norwich, NR4 7UQ

Email: R.Bundy@uea.ac.uk 


\begin{abstract}
Diet and physical activity play key roles in maintaining health and preventing or delaying disease. The global prevalence of dementia, an umbrella term describing a set of symptoms which affect cognitive function, is set to rise dramatically by 2050 , with current drug treatments inadequate. Diet and physical activity are modifiable factors in dementia, influencing both disease risk and pathophysiology. It has been known since the 1950s that a Mediterranean diet has positive health benefits. Over the past 25 years, a causal link has been shown between consumption of a Mediterranean diet and decreased risk of cardiovascular disease (CVD), particularly in Mediterranean countries. There have also been indications that a Mediterranean diet and increased physical activity can improve cognitive health and delay the onset of dementia. However, causative demonstrations of these effects, as well as the feasibility of implementing such lifestyle changes in a non-Mediterranean population, are lacking. The MedEx-UK (Diet, physical activity and dementia risk in UK adults) programme will attempt to address both these points.
\end{abstract}

\title{
Introduction
}

The World Health Organisation (WHO) defines dementia as a syndrome, usually progressive, where there is deterioration in cognitive function beyond what might be expected from normal ageing (WHO 2018). There are many dementia subtypes, with Alzheimer's disease and vascular dementia accounting for up to $80 \%$ of cases, and prevalence is rapidly increasing in the UK and worldwide (SACN 2018). Globally, around 50 million people currently have dementia, with nearly 10 million new cases every year, and there is a predicted tripling in prevalence to over 150 million by 2050 (WHO 2018). In the UK, the Alzheimer's Society note that 816,000 people were affected by dementia in the UK in 2014 (AS 2014), and two years later, it had overtaken ischemic heart disease to become the leading cause of death in England and Wales, accounting for 12\% of registered deaths (ONS 2017). The associated social and financial implications are huge, the latter suggested as $\$ 2$ trillion 
globally by 2030 (WHO 2018). Some authors have suggested that dementia represents the greatest global challenge for health and social care in the $21^{\text {st }}$ century (Livingston et al. 2017).

Encouragingly, age-specific incidence in dementia is declining, certainly in the UK, US and Sweden (Mathews et al. 2013), which may in part be attributable to improved cardiovascular health (Wu et al. 2016). Many of the key biological processes underlying the disease are potentially modifiable by behavioural intervention, such as with diet and physical activity (Deckers et al. 2015). A recent report noted that delaying the onset of dementia by 2 or 5 years would result in a $19 \%$ and $33 \%$ reduction in prevalence respectively by 2050 , and a much lower prevalence of severe dementia (Lewis et al. 2014). In the absence of a cure or effective drug intervention, and with some pharmaceutical companies unwilling to invest more money in this area, investigating the potential of modifiable factors such as diet and physical activity to delay progression of dementia is an urgent concern. Two recent reviews provide focus for future work. First, the UK Scientific Advisory Committee on Nutrition (SACN) statement entitled 'Diet, Cognitive Impairment and Dementias' (2018) concluded there is evidence of the specific benefits of a Mediterranean dietary pattern (MDP) on reducing the risk of dementia, although noted thatmore evidence is needed from randomised control trials (RCTs). Second, a recent Lancet Commission stated that of three mechanisms linked to dementia, adherence to a Mediterranean diet could positively influence two ('reduced brain inflammation' and 'reduced brain damage'), and physical activity could positively affect all three (the third being 'increased brain cognitive reserve') (Livingston et al. 2017).The MedEx-UK programme will specifically study the effects of a Mediterranean diet and physical activity on cognitive function in UK adults at above average risk of dementia.

\section{The Mediterranean diet pattern and health}

The benefits of what Ancel Keys described as a 'good Mediterranean diet' on serum cholesterol levels were first observed by him and his co-workers in Italy in the 1950s, and led to the subsequent publication of the seven countries study (Keys et al. 1995). Epidemiological evidence from large cohorts demonstrates protective effects of the Mediterranean diet against cardiovascular diseases 
(CVD), hypertension, obesity, cancer and all-cause mortality. Subsequently, RCTs have demonstrated benefits of the MDP for the primary and secondary prevention of CVD, type 2 diabetes, atrial fibrillation and breast cancer (Tosti et al. 2017).

The Mediterranean diet is defined as being rich in plant foods (cereals, fruit, vegetables, legumes, tree nuts, seeds and olives), with olive oil as the principal source of added fat. Also important are moderate to high intakes of fish and seafood with a correspondingly lower consumption of red meat, moderate consumption of eggs, poultry and dairy products, and a moderate intake of alcohol (mainly wine during meals). In addition, and sometimes forgotten, are considerations such as a preference for seasonality, fresh and minimally processed foods, conviviality (e.g. eating meals slowly in the company of others), as well as a lifestyle promoting regular physical activity and adequate rest (BachFaig et al. 2011).

In a recent review on the health benefits of the MDP, Tosti and colleagues (2017) suggested that the most important health-promoting mechanisms induced by a MDP are: a lipid-lowering effect; protection against oxidative stress, inflammation and platelet aggregation; modification of hormones and growth factors involved in cancer; the inhibition of nutrient sensing pathways by restricting specific amino acids; and gut microbiota-mediated production of metabolites. In addition, Shannon and colleagues (2018) also note the potential of a MDP to increase nitric oxide (NO) production, implicated in many complementary physiological processes such as blood pressure regulation, glucose homeostasis, and neurotransmission. Paradoxically, many of the good habits of the traditional Mediterranean diet and way of life first described by Keys in the 1950s are being lost in countries where they were first observed (e.g. Italy, Spain, Greece) with a corresponding increase in metabolic diseases such as CVDs and certain cancers (Tosti et al. 2017).

\section{Heart health and cognitive health}


The effect of a MDP on CVD risk was initially of great interest to Keys and other researchers, in light of the historical high rate of CVD observed in the US and Northern Europe compared to the Mediterranean countries. The Lyon Diet Heart study of the 1990s was the first RCT to show a protective effect of a MDP against CVD mortality in 605 patients who had previously suffered myocardial infarction (MI). So successful was the trial, it was stopped early after the intervention group showed a massive $73 \%$ reduction in the rate of cardiac deaths and non-fatal MIs combined compared to controls after 27 months (de Lorgeril et al. 1994). Subsequently, the multi-centre Prevención con Dieta Mediterránea (PREDIMED) study, a large randomised intervention of the effects of the Mediterranean diet supplemented with key ingredients, was published by a Spanish consortium of researchers (Estruch et al., 2013). Participants ( $n=7447)$ aged 55 to 80 years and at high risk of CVD were randomised into one of three study groups: Mediterranean diet supplemented with extra-virgin olive oil (EVOO, 1 litre per week); Mediterranean diet supplemented with nuts (30 g per day of mixed tree nuts); or a control group (given advice to reduce dietary fat based on American Heart Association recommendations). An approximate $30 \%$ reduction in the relative risk of major CV events was observed in both intervention groups compared with controls after a median 4.8 year follow-up [adjusted hazard ratios (HR) 0.70 (95\% CI: 0.54 to 0.92 ) and 0.72 (95\% CI: 0.54 to 0.96 ) for the EVOO and nuts groups respectively].

Such powerful effects of the Mediterranean diet on CVD outcomes have been followed by evidence on the potential of a Mediterranean diet to improve cognitive health, and delay or reduce the incidence of dementia. As mentioned previously, CVD and dementia share some underlying physiological processes, such as deregulated glucose and lipid metabolism, reduced vascular function and tissue perfusion, and a pro-inflammatory and oxidative state. It is interesting to note that in the PREDIMED study, stroke risk (a component of the primary outcome score) decreased significantly by almost $40 \%$ in both intervention groups combined. It is therefore likely that improved cardiovascular health, including increased cerebral blood flow, contribute to the improved cognition associated with a MDP. 
SACN has recently reviewed the epidemiological evidence from a number of meta-analyses and systematic reviews of the effects of a MDP on cognitive function and dementia (including a total of 19 prospective cohort studies in participants ranging from 62- to 80-years-old) and note that the majority of these studies show a beneficial association (SACN 2018). For example, a meta-analysis by Singh and colleagues (2014) noted that subjects with the greatest adherence to a MDP (highest versus lowest tertile) had a $27 \%$ reduced risk of developing mild cognitive impairment (MCI) [two prospective cohort studies (PCS): HR 0.73 (95\% CI: 0.56 to 0.96)], a $36 \%$ reduced risk of developing Alzheimer's disease (AD) [two PCS: HR 0.64 (95\% CI: 0.46 to 0.89)] and a 33\% reduced risk of developing either MCI or AD [four PCS: HR 0.67(95\% CI: 0.55 to 0.81)]. Cao et al. (2016) observed similar effects sizes, in a meta-analysis that included five PCS [3 of which were also included in Singh et al. (2014)] and which showed positive association between a MDP and reduced risk of developing cognitive impairment (MCI or dementia) [relative risk (RR) $0.69,95 \%$ CI: 0.57 to 0.84 ]. Psaltopoulou et al. (2013) pooled data from four PCSs, four cross-sectional studies and one casecontrol study in a meta-analysis which showed that high (but not moderate) adherence to a MDP was associated with less cognitive impairment (MCI, AD and other dementias; RR 0.60, 95\% CI: 0.43 to 0.83). Finally, Sofi et al. (2010) observed in a meta-analysis of four PCS that a two-point increase in a 9-point Mediterranean diet adherence score (of which a higher score shows better compliance to a MDP) was associated with lower risk of developing neurodegenerative diseases.

Of particular relevance to the planned $M e d E x-U K$ study, is a 14 year prospective cohort study comprising 1880 community-dwelling elders without dementia living in New York. This showed that both Mediterranean-type diet adherence [compared with low diet score, HR or high diet score was 0.60 (95\% CI: 0.42 to 0.87 )] and physical activity [compared with no physical activity, HR for high physical activity was 0.67 (95\% CI: 0.47 to 0.95 )] were associated with lower AD risk (Scarmeas $e t$ al. 2009). Compared with those neither adhering to the diet nor participating in physical activity, volunteers with the highest adherence to a MDP and high physical activity had a lower risk of AD [HR, 0.65 (95\% CI: 0.44 to 0.96$)]$. 
The only RCT included by SACN on the effect of a MDP on cognitive health is a sub-study of the original PREDIMED trial from the Navarra cohort (Martinez-Lapiscina 2013a). This showed small but significant benefits in both Mediterranean diet intervention groups compared with the control group for the Mini-mental State Examination [MMSE; HR 0.62 (95\% CI: 0.18 to1.05) for the EVOO group and HR 0.57 (95\% CI 0.11 to 1.03 ) for the nuts group, of a 30 point scale] for the clock drawing test, a simple tool that is used to screen people for signs of neurological problems [HR 0.51 (95\% CI: 0.20 to 0.82 ) for the EVOO group and HR 0.33 (95\% CI: 0.003 to 0.67 ) for the nuts group, of a 7 point scale] in 522 adults over 6.5 years. Additionally, MCI was assessed in a sub-cohort of the same trial $(n=268)$ and a reduced risk was observed in the Mediterranean diet group supplemented with EVOO [OR 0.34 (95\%: CI: 0.12 to 0.97 ), but not in the group supplemented with nuts (MartinezLapiscina 2013b). Notably, cognitive outcomes were only measured at the end of the follow-up period, and so differences may have been present at baseline (SACN 2018)

A recent systematic review, however, identified five separate RCTs on the effects of a MDP on cognition, including five specific sub-studies from PREDIMED RCT (only two of which were reported by SACN; Radd-Vagenas et al. 2018). The authors note that only 8 of the 66 cognitive outcomes measured within these studies indicated that Mediterranean diet was associated with better cognitive performance, with effect sizes ranging from small (0.32) to large (1.66). They concluded that although there is inconsistent evidence for an overall significant effect of a Mediterranean diet on cognitive function, evidence from the five PREDIMED sub-studies (which were deemed to be the best designed) was strongest. In these trials, three composite cognitive scores (memory, frontal and global function) were found to be significantlyimproved by a MDP, with effect sizes ranging from 0.39 to 1.29. This led the authors to suggest that additional research from well-designed RCTs is warranted.

\section{Future directions and MedEx-UK}


While attempts have been made to disentangle the effects of specific components of the Mediterranean diet and their effects on health (which include mono- and poly-unsaturated fatty acids, such as oleic and omega-3s, soluble fibre, vitamins and minerals, and various polyphenols), intervention with a portfolio diet approach, such as a MDP, provides a more pragmatic approach from a public health perspective. SACN has concluded there is currently insufficient evidence linking individual nutrients (B vitamins, vitamins $\mathrm{C}$ and $\mathrm{E}$ and omega-3 fatty acids and polyphenols) with prevention of cognitive decline or cognitive impairment, and other authors have suggested synergistic and interactive effects of various nutrients found in the MDP on reducing inflammation (Tosti et al. 2017).

There has been recent interest in using multi-domain interventions as an approach to improve health outcomes. A recent example specific to dementia is the Finnish Geriatric Intervention Study to Prevent Cognitive Impairment and Disability (FINGER) trial, which assessed the effect of a diet based on Finnish Nutrition Recommendations, exercise, cognitive training and monitoring of vascular risk in at-risk elderly Finnish adults over a 2-year period, and reported an effect size of 0.13 on cognitive performance (Ngandu et al. 2015). Approaches which specifically support behavioural modification to improve adherence to diet or exercise may also improve the chances of a successful intervention. For example, the PREDIMED programme provided both individual (with a dietitian) and group support for participants approximately every 3 months, meaning between 12 and 26 sessions of each depending on the overall study length, and this robust-design may have contributed in part to the success of the programme (Raad-Vagenas et al. 2018).

Finally, SACN (2018) and Raad-Vagenas and colleagues (2018) have made recommendations for future research in the area of diet and cognition. A summary of salient points include: having more evidence from RCTs, targeting at-risk groups; using a parallel design to avoid potential residual changes in cross-over studies; characterising relevant genotypes and lifestyle factors related to cognition; using various tests of cognition and brain morphology; assessing biomarkers and 
neuroimaging; baseline assessment of the Mediterranean diet; defining minimum quantities of recommended foods; providing culinary instructions (including cooking); controlling cohorts for relevant confounding factors, such as childhood cognitive ability; and using novel technologies to increase compliance, such as behavioural strategies.

MedEx-UK, funded by Alzheimer's Research UK (ARUK) to run initially between January 2018 and June 2020, is the first multi-domain programme in the UK to study to effects of a MDP and physical activity on cognitive health. The first intervention phase of the programme is to run a feasibility study of changing diet and physical activity over a 24-week period (during 2019), with the intention to conduct a follow-on intervention study over a 2- to 5-year period to assess the effect of a MDP and physical activity on cognitive decline in 1000+ adults at above average risk of dementia. Previous evidence has shown that adhering to a MDP in a non-Mediterranean country such as the UK can present certain difficulties, such as adapting to a new eating pattern, and a non-conducive British culture and climate, presenting potential barriers to long-term adherence (Middleton et al. 2015). Therefore, it is important to assess how effectively people's behaviour can be changed before upscaling the intervention.

For the feasibility trial, the aim is to recruit 108 adults aged 55-74 years with a CVD risk profile (a QRISK3 score of 15 or above) and subjective memory impairment, who are therefore at above average risk of dementia. The study is multi-centre, being conducted at UEA (UK), Birmingham University (UK), and Newcastle University (UK), where recruitment will be through local general practitioners. Potential participants will be initially screened using an online questionnaire. The main recruitment criteria are a Mediterranean diet score of less than nine (based on the same 14-point Mediterranean Diet Questionnaire employed by PREDIMED), and physical activity levels less than 100 minutes per week, with exclusions for those who meet dementia or MCI criteria, or who have moderate to severe depression, or any other serious mental disorder. Participants will be randomised onto one of three arms: MDP, MDP plus physical activity, and the control group. 
$M E D E x-U K$ will have a strong behavioural intervention component to help effect change. First, a comprehensive online platform called LEAP2 will be used, previously developed for the LiveWell study (Lara et al. 2016), which will allow participants to search for Mediterranean style recipes, plan and log their physical activity levels, be sent and receive online support, and shop (through a supermarket online delivery system) for Mediterranean style ingredients. The second component will be group-based interventions, consisting of group sessions, which will involve training on the online platform, explanation of the principles of the Mediterranean diet and physical activity requirements, peer support to identify and overcome barriers to adopting a Mediterranean diet, as well as providing ideas and support to adhere to it. The control group will be provided with advice on healthy eating. They will have basic access to the online platform, but no group sessions beyond initial training.

The primary outcome for this feasibility study will be the ability of participants to increase their Mediterranean diet score by at least 3 points and physical activity to over 150 minutes of moderate activity per week. Secondary outcomes include a battery of cognitive function tests and magnetic resonance imaging to examine functional and temporal changes in brain structure, vascular assessments including flow mediated dilation, various plasma biochemical markers and APOE genotyping. Although the feasibility study will be underpowered to examine the impact of intervention on many of these outcome measures, their inclusion is to inform the design of a larger follow-on MedEx-UK intervention trial.

\section{Conclusion}

MedEx-UK is a multi-domain programme that will study the effect of a MDP and physical activity on cognitive health, and will address many of the shortfalls of previous research, by having a robust study design, appropriate measures of diet and cognition, and behavioural intervention to increase compliance. If the feasibility trial is successful, a larger follow-up study will be undertaken, which 
could eventually provide a structured and systematic approach to reducing dementia risk in at-risk adults using diet and exercise with group and peer support. As mentioned previously, a shift in the onset of dementia by even 2 years would have a significant effect on prevalence in the UK population with reduced burden on the NHS, as well as society in general.

\section{Acknowledgements}

The current project 'Diet, physical activity and dementia risk in UK adults: Epidemiology and MedEx feasibility study' is funded by the Alzheimer's Research UK Prevention and Risk Reduction Fund, reference number ARUK-PRRF2017-006UK. Co-investigators on the MedEx-UK programme are Professor Michael Hornberger (UEA), Dr Narelle Berry (UEA), Dr Wendy Hardeman (UEA), Dr Sarah Hanson (UEA), Professor John Mathers (Newcastle University), Dr Blossom Stephen (Newcastle University), Dr Mario Siervo (Newcastle University), Dr Stella Paddick (Newcastle University), Dr Sarah Aldred (Birmingham University) and Professor Hugh Markus (Cambridge University).

\section{Conflict of interest}

The authors have no conflict of interest to declare

\section{References}

AS (Alzheimer's Society) (2014) Dementia UK: Update, London: Alzheimer's Society

Bach-Faig A, Berry EM, Lairron D et al. (2011) Mediterranean diet pyramid today. Science and cultural updates. Public Health Nutrition 14: 2274-2284

Cao L, Tan L, Wang HF, Jiang T et al. (2016) Dietary Patterns and Risk of Dementia: a Systematic Review and Meta-Analysis of Cohort Studies. Molecular Neurobiology 53: 6144-6154 
Deckers K, van Boxtel MP, Schiepers OJ et al. (2015) Target risk factors for dementia prevention: a systematic review and Delphi consensus study on the evidence from observational studies. International Journal of Geriatric Psychiatry 30: 234-246

de Lorgeril M, Renaud S, Mamelle N, et al. (1994) Mediterranean alpha-linolenic acid-rich diet in secondary prevention of coronary heart disease. Lancet 343:1454-1459

Estruch R, Ros E, Salas-Salvadó J, et al. (2013) PREDIMED Study Investigators. Primary prevention of cardiovascular disease with a Mediterranean diet. New England Journal of Medicine 368:12791290.

Keys A (1995) Mediterranean diet and public health: Personal reflections. The American Journal of Clinical Nutrition. 61:1321S

Lara, J., et al. (2016) Pilot Randomised Controlled Trial of a Web-Based Intervention to Promote Healthy Eating, Physical Activity and Meaningful Social Connections Compared with Usual Care Control in People of Retirement Age Recruited. PLoS One 11: p. e0159703.

Lewis F, Karlsberg Schaffer S, Sussex J et al. (2014) The Trajectory of Dementia in the UK - Making a Difference. Office of Health Economics: Consulting Report https://www.ohe.org/publications/trajectory-dementia-uk-making-difference (Accessed 4/5/2018)

Livingston G, Sommerlad A, Orgeta V et al. (2017) Dementia prevention, intervention, and care. Lancet 390: 2673-2734

Martinez-Lapiscina EH, Clavero P, Toledo E et al. (2013a) Mediterranean diet improves cognition: 
the PREDIMED-NAVARRA randomised trial. Journal of Neurology, Neurosurgery, and Psychiatry $84: 1318-1325$

Martinez-Lapiscina EH, Toledo E, Clavero P et al. (2013b) Virgin olive oil-rich Mediterranean diet improves cognition: the PREDIMED-Navarra randomized, prevention trial. Annals of Nutrition \& Metabolism 62:36

Matthews FE, Arthur A, Barnes LE et al. (2013) A two-decade comparison of prevalence of dementia in individuals aged 65 years and older from three geographical areas of England: results of the Cognitive Function and Ageing Study I and II. Lancet 382:1405-12

Middleton, G. et al. (2015) Brief Report: Implementing a Mediterranean Diet Intervention into a RCT: Lessons Learned from a Non-Mediterranean Based Country. The Journal of Nutrition Health and Aging 19:1019-1122.

ONS (Office for National Statistics) (2017) Deaths registered in England and Wales (Series DR): 2016, UK: Office for National Statistics.

https://www.ons.gov.uk/peoplepopulationandcommunity/birthsdeathsandmarriages/deaths/datasets/de athsregisteredinenglandandwalesseriesdrreferencetables (Accessed 4/5/2018)

Psaltopoulou T, Sergentanis TN, Panagiotakos DB et al. (2013) Mediterranean diet, stroke, cognitive impairment, and depression: A meta-analysis. Annals of Neurology: 74: 580-591

Radd-Vagenas SR, Duffy SL, Naismith SL et al. (2018) Effect of the Mediterranean diet on cognition and brain morphology and function: a systematic review of randomized controlled trials. American Journal of Clinical Nutrition 107: 389-404 
SACN (Scientific Advisory Committee on Nutrition) (2018) Statement on Diet, Cognitive Impairment and Dementias

https://assets.publishing.service.gov.uk/government/uploads/system/uploads/attachment_data/file/685

153/SACN_Statement_on_Diet_Cognitive_Impairment_and_Dementias.pdf. (Accessed 4/5/2018)

Scarmeas N, Luchsinger JA, Schupf N et al. (2009) Physical activity, diet, and risk of Alzheimer disease. JAMA 302:627-37

Shannon OM, Stephan BCM, Minihane AM3 et al. (2018) Nitric oxide boosting effects of the Mediterranean diet: A potential mechanism of action. The Journals of Gerontology. Series A, Biological Sciences and Medical Sciences. Apr 19. [Epub ahead of print]

Singh B, Parsaik AK, Mielke MM et al. (2014) Association of mediterranean diet with mild cognitive impairment and Alzheimer's disease: a systematic review and meta-analysis. Journal of Alzheimer's Disease 39: 271-282

Sofi F, Abbate R, Gensini GF et al. (2010) Accruing evidence on benefits of adherence to the Mediterranean diet on health: an updated systematic review and meta-analysis. American Journal of Clinical Nutrition 92: 1189-1196

Tosti V, Bertozzi B, and Fontana, L (2017) Health Benefits of the Mediterranean diet: metabolic and molecular mechanisms. The Journals of Gerontology. Series A, Biological Sciences and Medical Sciences 73: 318-326

WHO (World Health Organisation) (2018) Dementia: key facts. http://www.who.int/en/newsroom/fact-sheets/detail/dementia. (Accessed 4/5/2018) 
Wu YT, Fratiglioni L, Matthews FE et al. (2016) Dementia in western Europe: epidemiological evidence and implications for policy making. Lancet Neurology 15:116-124 